\title{
Ventricular Tachycardia After Naloxone Administration: a Drug Related Complication? Case Report and Literature Review
}

\author{
Heleen Lameijer • Nasim Azizi • Jack J. M. Ligtenberg • \\ Jan C. Ter Maaten
}

(C) The Author(s) 2014. This article is published with open access at Springerlink.com

\begin{abstract}
Objective To present a case of ventricular tachycardia following naloxone administration and to review current literature concerning ventricular tachycardia associated with naloxone.

Methods We present a case and review the literature concerning ventricular tachycardia (VT) as a complication of naloxone administration.

Results In our patient, a 44-year old male intoxicated multi-drug user, VT presented on the electrocardiogram shortly after naloxone (twice $0.4 \mathrm{mg}$ intra-osseous) administration for suspected methadone overdose. After initial medical treatment he was treated with electro cardioversion because of hemodynamic instability. Our patient was subsequently stabilized and admitted to our intensive care unit (ICU). Eight comparable cases concerning VT after administration of naloxone were found in the literature, both in multi-drug uses as in patients receiving opiates for elective surgery.

Conclusion We suggest VT as a possible, but rarely reported serious complication of naloxone administration (Naranjo scale possible to probable). Patients who are multi-drug users or receive opiates in high doses may be prone to VT/VF due to acute (iatrogenic) opiate withdrawal or reduction of sympathetic suppression and therefore
\end{abstract}

Written informed consent was obtained from the patient for publication of this case report. A copy of the written consent is available for review by the Editor-in-Chief of this journal.

H. Lameijer $(\varangle) \cdot$ N. Azizi · J. J. M. Ligtenberg ·

J. C. Ter Maaten

Department of Emergency medicine, University Medical Centre

Groningen (UMCG), University of Groningen, Hanzeplein 1,

9700 RB Groningen, The Netherlands

e-mail: h.lameijer@umcg.nl overstimulation. Also, antagonism of the protective mechanism of opioids against sympathetic excess (due to substance abuse, cardiac disease or hypoxia, as seen in all cases) may induce VT/VF. We suggest the use of small dosages $(0.1 \mathrm{mg}$ vs $0.4 \mathrm{mg}$ ), cardiac monitoring, and to have defibrillation devices stand-by.

\section{Key Points}

Ventricular tachycardia is suggested to be a possible but rarely reported complication of naloxone administration.

Multi-drug users or patients receiving iatrogenic high opiate doses may be prone to naloxone induced ventricular tachycardia.

We suggest the use of small dosages, cardiac monitoring, and to have defibrillation devices standby when using naloxone in selected patients.

\section{Introduction}

Administration of naloxone, an opioid receptor antagonist frequently used in overdose with morphine (iatrogenic) or heroin, may not be as safe as previously considered. Known adverse events of naloxone administration are nausea, dizziness, headache and, in frequent opiate users, acute (iatrogenic) opiate withdrawal syndrome. We describe a patient who develops a ventricular tachycardia (VT) after administration of naloxone. Furthermore, we review the literature concerning VT following the use of naloxone. 


\section{Case Report}

A 44 year old former intravenous drug addict was brought by ambulance to our emergency department with a reduced state of consciousness. His medical history reveals hay fever, chronic hepatitis C infection (hepatitis B and HIV negative) and arthritis of his right knee. Furthermore, an atrial septal defect had been discovered two years ago and was surgically corrected. Being a former drugs addict, he uses methadone $70 \mathrm{mg}$ once a day, diazepam $10 \mathrm{mg}$ three times a day and promethazine $25 \mathrm{mg}$ once a day if needed. He uses no cardiovascular medication. Because of his reduced mental state and his medical history a methadone overdose or relapse in intravenous drug use, hence opioid overdose, was suspected and naloxone (twice $0.4 \mathrm{mg}$ intraosseous) was given following protocol by ambulance personnel before presentation at our emergency ward.

On arrival our patient was agitated but able to speak. His airway was clear. His breathing was compromised with a frequency of 40 breaths per minute (normal 14-20 per minute) and normal breath sounds. Because of agitation measuring saturation in this patient was difficult and therefore unreliable. Blood gas analysis showed a respiratory alkalosis with normal oxygen pressures $(\mathrm{pH} 7.51$ (7.35-7.45); pCO2 $2.5 \mathrm{kPa}$ (4.6-6.0); $\mathrm{pO} 2 \quad 11.9 \mathrm{kPa}$ (9.5-13.5) HCO3 $15 \mathrm{mmol} / \mathrm{l}(21-25)$ ), oxygen saturation $98 \%$ without oxygen administration. Because of tachypnea he received a non-rebreathing mask with $151 \mathrm{O}_{2} / \mathrm{min}$. He showed a blood pressure of $140 / 78 \mathrm{mmHg}$, a tachycardia of 160-180 beats per minute (normal $60-100 \mathrm{bpm}$ ) and a delayed capillary refill of $5 \mathrm{~s}$ (normal $\leq 2 \mathrm{~s}$ ). No murmurs were heard at cardiac auscultation. Electrocardiography shows frequent episodes of VT. His Glascow coma score is reduced (4-4-2, normal 4-6-5), both pupils were dilated. His temperature was 37 degrees Celsius, but rose to 39.6 degrees Celsius at secondary assessment half an hour later. Additional laboratory results showed Calcium level of

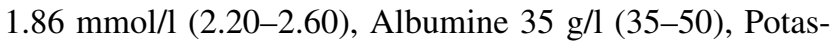
sium $4.7 \mathrm{mmol} / \mathrm{l}$ (3.5-5.0) and Magnesium 0.97 $(0.700-1.00)$. We concluded our patient to be in distributive or cardiac shock. He received 2,250 mg Magnesiumsulphate for his VT. Amiodarone $300 \mathrm{mg}$ was given because of sustained VT, without sufficient effect. Because of progressive agitation despite administration of midazolam $5 \mathrm{mg}$, increased frequency of breathing and difficulty in measuring oxygen pressures because of agitation, he was sedated, using propofol and roccuronium, and intubated. Additionally, an arterial tube was placed in the brachial artery for frequent blood gas analysis. A sudden fall in blood pressure made us decide to perform electro cardioversion for VT (200 J), which restored sinus rhythm. Our patient was subsequently stabilized and admitted to our intensive care unit (ICU). Myocardial infarction was ruled out by serial electrocardiograms and the pattern of serial cardiac enzymes. We contributed the initial rise in temperature to rhabdomyolysis due to agitation, which developed further during hospital stay (maximal CK-levels 91,716 U/1, normal 0-200 U/l; Myoglobin in urine $575 \mathrm{ug} / \mathrm{l}$, normal 0-21 ug/l). Rhabdomyolysis was treated with intravenous fluid administration and forced diuresis. Because of mixed sympathomimetic and opioid toxidrome at presentation in our ward, multiple toxicology tests were performed once our patient was stabilized. These showed signs of both amphetamines, cannabis, benzodiazepine, opiate, methadone and cocaine.

\section{Literature Review}

Methods

We searched the MedLine database for Naloxone OR Narcan AND ventricular tachycardia, Naloxone OR Narcan AND "Tachycardia, Ventricular"[Mesh], Naloxone OR Narcan AND ventricular fibrillation, Naloxone OR Narcan AND "Ventricular Fibrillation"[Mesh], Naloxone OR Narcan AND sudden cardiac death, Naloxone OR Narcan AND "Death, Sudden, Cardiac"[Mesh]. Date last searched was 13-02-2014. Articles were excluded based on abstract and title; studies describing only supraventricular arrhythmias, stress cardiomyopathy without cardiac arrhythmias, animal or in vitro studies were excluded. Articles not online available or directly available for the University Medical Centre Groningen were bought. Duplicates were removed. Articles considered significant were included by cross referencing. In addition, we searched the Dutch and European public pharmacovigilance databases (LAREB and Eudravigilance) for $\mathrm{VT}$ as a side effect of naloxone administration.

\section{Results}

Only 7 studies describing 8 cases were found matching our criteria, dating from 1974 to 2005. The Dutch and European public pharmacovigilance databases have not reported VT as a side effect of naloxone administration. One report described VT in a patient using Nalorphine. For comparison, see Table 1. The patient described by Cuss et al. suffered VT shortly after administration of Naloxone [2]. A second gift resulted in a second VT. Later, when readmitted, he again suffered VT twice, after 30 and $50 \mathrm{~min}$. Two patients died, one due to VT, one due to asystole.

\section{Discussion}

We reviewed the current literature concerning VT/VF following the use of naloxone and contribute to this 


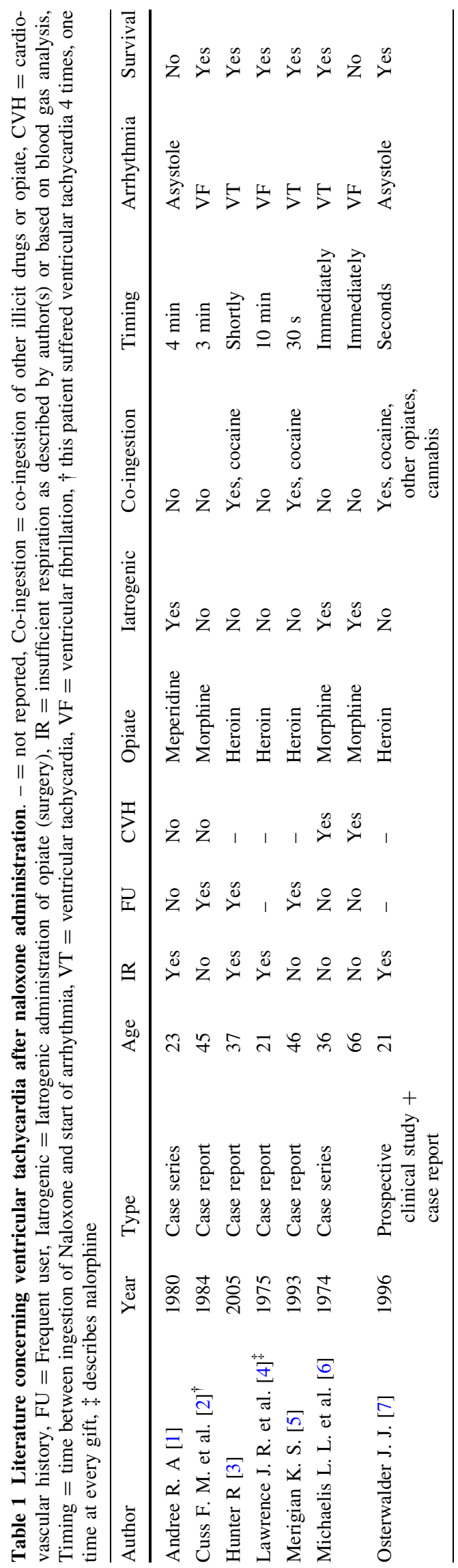

literature presenting our own case. The fact that we only found 8 cases matching our search criteria indicated rarity or underreporting. Of these, 6 described VT or ventricular fibrillation (VF) following naloxone administration. Three out of six patients suffering VT/VF were reported to be frequent drug users $(50 \%)$, as was our patient. The use of an opiate antagonist in frequent drug use may increase the chance of acute opiate withdrawal syndrome, complicated by VT/VF. Another two patients received high dose morphine for cardiac surgery, which may as well trigger acute opiate withdrawal syndrome when opiate antagonists are used.

Our patient could have been more prone to arrhythmogenic events due to (late discovery and correction of) his congenital heart disease and therefore structural cardiac changes. Only two patients had a history with cardiac disease, therefore susceptibility of the myocardium for arrhythmogenic events could not be a sufficient explanation for VT/VF in these patients.

Cocaine, as used by our patient, is known for its arrhythmogenic and ischemic complications, and could have contributed to the VT in our patient [8]. Cocaine was used by only two of the other patients who suffered from VT/VF; multi drug use was seen in both patients. Cocaine alone, multi-drug use or an interaction with naloxone, can therefore not be solely responsible for VT/VF in all of these patients, and contribution of naloxone to the VF/VF could be suspected.

At presentation our patient showed signs of insufficient respiration. It had previously been suggested that VT following the use of naloxone could have been triggered by hypoxia alone and not by administration of naloxone itself $[1,4,9]$. However, only 2 out of 6 patients with VT/VF had signs of insufficient ventilation in this review. Notably, in all cases including our case VT/VF starts shortly after administration of naloxone. However, short duration of this interval suggests an association in these, cases, not per se causality. The repeatability of VT in the case of Cuss et al. [7] suggests an association as well, but could also be attributed to ventricular irritability due to other mechanisms in this patient despite lack of cardiac history.

Naloxone is suggested to increase or overstimulate sympathetic activity by suddenly reducing the sympathetic suppression of the administered opiate and therefore increase the risk on VT/VF [2, 7]. Laboratory assessments in dogs on morphine show only increased rate of ventricular extra systoles after naloxone administration, but not clearly increased ventricular irritability [6]. In all cases sympathetic excess may have been caused by different mechanisms, such as hypoxia, substance abuse or cardiac disease. This combined with a sudden loss of the opioid induced protection against arrhythmias due to naloxone administration may have triggered sympathetic overdrive 
in these patients. In addition, minor corrected QT interval prolongation due to relatively high dose naloxone infusion (40 ug/ $/ \mathrm{kg} / \mathrm{min}$ ), and therefore possible susceptibility for cardiac arrhythmias, has been described in a very small population [10].

Worldwide accepted protocol administration of naloxone directs dosing of $0.4 \mathrm{mg}$, which may be repeated for insufficient response. Smaller dosages have been used in controlled settings (during anesthesiology) with sufficient response. Starting with a dose of $0.1 \mathrm{mg}$ and increasing the dose slowly if needed may be the solution to prevent iatrogenic acute opiate withdrawal and therefore VT/VF in these patients [11]. Another solution could be to abandon administration of naloxone in opiate addicts, therefore acute withdrawal syndrome cannot take place, and have them intubated until awoken. However, this implicates higher healthcare costs due to hospital and ICU stays and should only be considered in patients at high risk for acute opiate withdrawal syndrome (as multi-drug users or patients who receive opiates in high doses). In other patients the lifesaving potential of naloxone overweighs the assumed risks, however, physicians need to be aware of them.

\section{Conclusion}

We suggest that VT/VF may possibly (Naranjo scale, even probable if previous reports are taken into account) be a serious complication of naloxone administration [12]. Patients who are multi-drug users or receive opiates in high doses may be prone to VT/VF due to acute (iatrogenic) opiate withdrawal or reduction of sympathetic suppression and therefore overstimulation. Also, antagonism of the protective mechanism of opioids against sympathetic excess (due to substance abuse, cardiac disease or hypoxia) may induce VT/VF. We propose to be careful with naloxone administration in these patients, and possibly choose not to use naloxone at all in this selected group of patients. If you do decide to administer naloxone, consider using small dosages $(0.1 \mathrm{mg}$ vs $0.4 \mathrm{mg})$ and have defibrillation devices stand-by. More research would help to determine the frequency and exactness of this possible complication, because, while highly interesting, current literature is scarce, evidence is little and our assumptions are made based on small proportions. However, ethical objections may preclude administration of naloxone in healthy individuals to prove causality for VT/VF as a complication of this opiate antagonist. Awareness and thereby more frequent reporting of this adverse event might also be important.

Acknowledgments Heleen LAMEIJER, MD: No funding, no financial interests, no conflicts of interest.

Nasim AZIZI, MD: No funding, no financial interests, no conflicts of interest.

Jack J.M. LIGTENBERG, MD PhD: No funding, no financial interests, no conflicts of interest.

Jan C. TER MAATEN, MD PhD: No funding, no financial interests, no conflicts of interest.

Open Access This article is distributed under the terms of the Creative Commons Attribution Noncommercial License which permits any noncommercial use, distribution, and reproduction in any medium, provided the original author(s) and the source are credited.

\section{References}

1. Andree RA. Sudden death following naloxone administration. Anesth Analg. 1980;59(10):782-4.

2. Cuss FM, Colaco CB, Baron JH. Cardiac arrest after reversal of effects of opiates with naloxone. Br Med J (Clin Res Ed). 1984;288(6414):363-4.

3. Hunter R. Ventricular tachycardia following naloxone administration in an illicit drug misuse. J Clin Forensic Med. 2005;12(4):218-9.

4. Lawrence JR, Lee FR. Letter: Ventricular fibrillation after narcotic withdrawal. Lancet. 1975;2(7937):717.

5. Merigian KS. Cocaine-induced ventricular arrhythmias and rapid atrial fibrillation temporally related to naloxone administration. Am J Emerg Med. 1993;11(1):96-7.

6. Michaelis LL, Hickey PR, Clark TA, Dixon WM. Ventricular irritability associated with the use of naloxone hydrochloride. two case reports and laboratory assessment of the effect of the drug on cardiac excitability. Ann Thorac Surg. 1974;18(6):608-14.

7. Osterwalder JJ. Naloxone-for intoxications with intravenous heroin and heroin mixtures-harmless or hazardous? A prospective clinical study. J Toxicol Clin Toxicol. 1996;34(4):409-16.

8. Kloner RA, Hale S, Alker K, Rezkalla S. The effects of acute and chronic cocaine use on the heart. Circulation. 1992;85(2):407-19.

9. Clarke SF, Dargan PI, Jones AL. Naloxone in opioid poisoning: Walking the tightrope. Emerg Med J. 2005;22(9):612-6.

10. Oldroyd KG, Rankin AC, Gray CE, Harvey K, Borland W, Rae $\mathrm{AP}$, et al. Failure to reproduce the in vitro cardiac electrophysiological effects of naloxone in humans. Br J Clin Pharmacol. 1994;37(3):289-94.

11. Bartlett D. The coma cocktail: Indications, contraindications, adverse effects, proper dose, and proper route. J Emerg Nurs. 2004;30(6):572-4.

12. Naranjo CA, Busto U, Sellers EM, et al. A method for estimating the probability of adverse drug reactions. Clin Pharmacol Ther. 1981;30(2):239-45. 